\title{
The Apelin Receptor APJ in Hematopoietic Stem Cells/ Progenitor Cells in the Early Stage of Non-Alcoholic Steatohepatitis
}

\author{
Wataru Ando ${ }^{a}$, Hiroaki Yokomori ${ }^{\mathrm{b}, \mathrm{d}}$, Katsuya Otori ${ }^{\mathrm{a}}$, Masaya Oda ${ }^{\mathrm{c}}$
}

\begin{abstract}
Background: Non-alcoholic steatohepatitis (NASH) is characterized by hepatic steatosis and inflammation with or without fibrosis. The apelin receptor (APJ) is related to angiotensin-like-receptor 1 (AGTRL1). The present study aimed to evaluate APJ as an indicator of the pathophysiology of early-stage NASH.
\end{abstract}

Methods: APJ expression was evaluated in six tissue samples with histologically proven early-stage NASH using immunohistochemistry (IHC) and immunoelectron microscopy (IEM).

Results: On IHC, in control liver tissue, APJ was mainly localized in the aortic artery, although APJ was detected only slightly in the sinusoids. In early NASH liver tissue, on IEM, APJ was observed mainly at the sites of sinusoidal lining cells around the central vein and periportal regions, and at arterial capillaries in the portal tract. With regard to endothelial cells (ECs), one sample showed a hematopoietic stem cell (HSC)/progenitor cell (HPC) partially wrapped with an EC.

Conclusion: HSCs/HPCs expressing APJ may contribute to the angiogenesis of liver tissue in early-stage NASH.

Keywords: APJ; Hematopoietic stem cell; Non-alcoholic steatohepatitis; Immunohistochemistry; Immunoelectron microscopy

\section{Introduction}

The formation of blood vessels is initiated by the assembly of

\footnotetext{
Manuscript submitted June 12, 2017, accepted June 29, 2017

aDepartment of Clinical Pharmacy, School of Pharmacy, Kitasato University, Tokyo, Japan

bepartment of Internal Medicine, Kitasato University Medical Center, Saitama, Japan

${ }^{\mathrm{c}}$ Departments of Internal Medicine, Sanno Hospital, International University of Health and Welfare, Tokyo, Japan

${ }^{\mathrm{d} C o r r e s p o n d i n g ~ A u t h o r: ~ H i r o a k i ~ Y o k o m o r i, ~ D e p a r t m e n t ~ o f ~ I n t e r n a l ~ M e d i c i n e, ~}$ Kitasato University Medical Center, Saitama 364-8501, Japan.

Email: yokomori@insti.kitasato-u.ac.jp
}

doi: https://doi.org/10.14740/jocmr3103w endothelial cells (ECs), which is termed vasculogenesis, and this is followed by angiogenesis, which results in the emergence of new vessels [1]. In both processes, to maintain the structural stability of nascent EC tubes, mural cells (MCs), such as smooth muscle cells and pericytes (PCs), are recruited around the forming tubes and adhere to ECs.

Apelin receptor (APJ), a ligand for angiotensin receptorlike 1 (AGTRL1), was recently isolated as a bioactive peptide from bovine gastric extract $[2,3]$. APJ is a G protein-coupled receptor, reportedly expressed in the pathophysiology of the cardiovascular system [3]. During angiogenesis, hematopoietic stem cells (HSCs)/progenitor cells (HPCs) migrate into avascular areas before ECs; therefore, angiopoietin-1 from these cells can induce angiogenesis by promoting EC chemotaxis [4]. Moreover, HSCs/HPCs regulate caliber size modification of blood vessels via APJ [4].

Non-alcoholic steatohepatitis (NASH) is characterized by hepatic steatosis and inflammation and liver cell damages [5]. The present study aimed to evaluate APJ as an indicator of the pathophysiology of early-stage NASH. We believe that the findings will help in the treatment of NASH.

\section{Materials and Methods}

Six patients with histologically confirmed Brunt classification stage 1, F1, early-stage NASH [5], seven patients with non-alcoholic fatty liver disease (NAFLD), and five normal controls were examined. Normal liver tissues were obtained from patients who had undergone hepatic resection because of metastasis of colon cancer. The antibodies for APJ were antiapelin receptor (APJ) rabbit polyclonal antibodies (MBL International, Woburn, MA, USA). We performed immunohistochemistry (IHC), and immunoelectron microscopy (IEM). The details of these methods have been described previously [6]. This study was approved by the relevant ethics committees of the institutions involved. Informed consent was obtained from all subjects (Supplementary 1, www.jocmr.org).

\section{Results}

On IHC, in control liver tissue, APJ was mainly localized in the hepatic artery, although APJ was detected only slightly in the sinusoids (Fig. 1a, b). In early NASH liver tissue, APJ was 

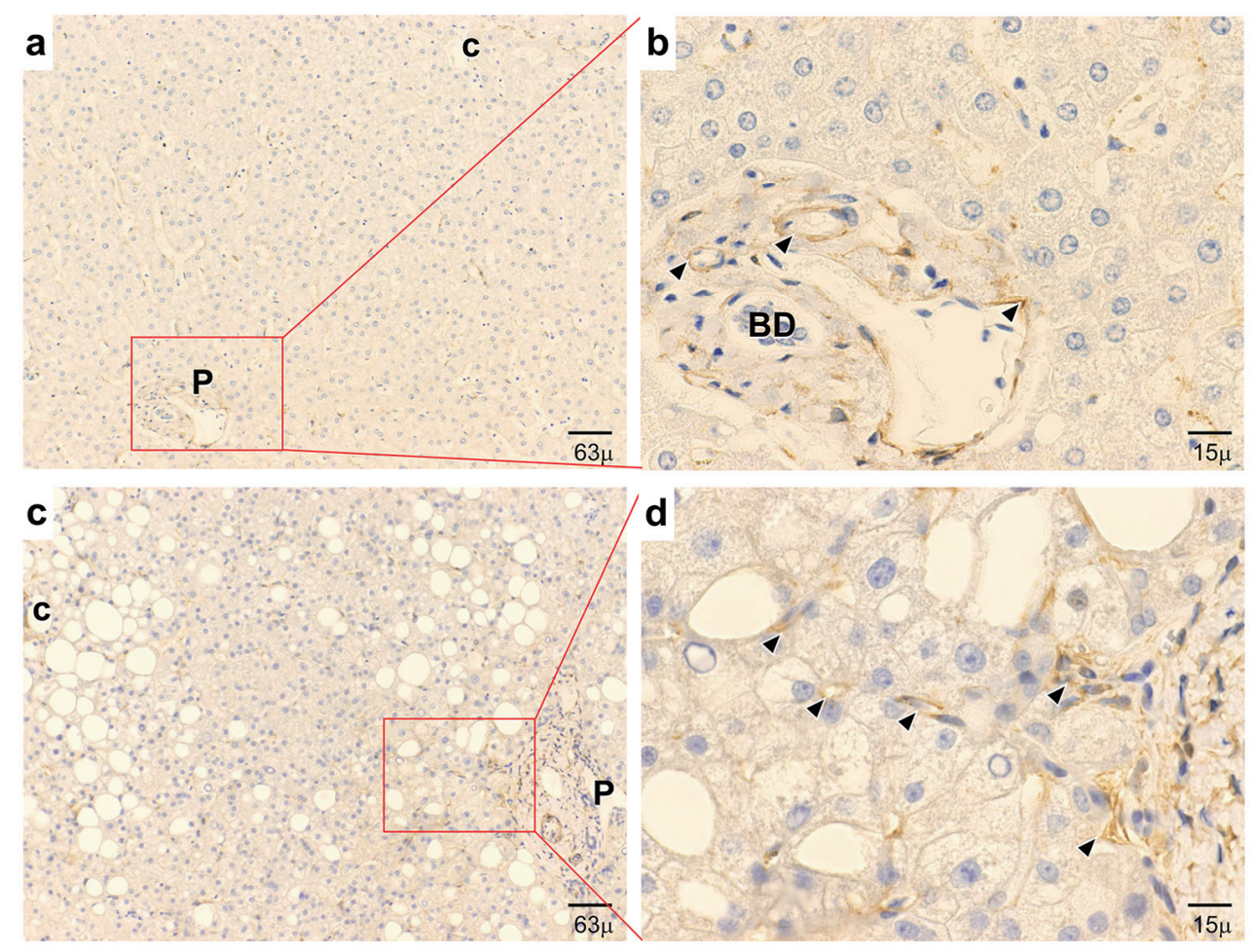

Figure 1. Immunohistochemical expression of APJ. (a, b) In normal control liver, APJ shows positive staining at vascular regions and capillaries in the periportal area. (c, d) In early-stage NASH liver, APJ shows strong positive staining at hepatic sinusoidal lining cells and inflammatory cells in the pericentral area and positive staining at hepatic sinusoidal lining cells in the periportal area. P: portal tract; c: central vein; BD: bile duct. $(a, c)$ Low magnification. (b, d) High magnification.

observed mainly at the sites of sinusoidal lining cells around the central vein and periportal regions, and at arterial capillaries in the portal tract (Fig. 1c, d). With regard to ECs, one sample showed an HSC/HPC partially wrapped with an EC (Fig. 2a, b). In another sample, only one region of a PC was attached to an EC (Fig. 2a, c). IEM showed the expression of APJ in both HSCs/HPCs and ECs and PCs and ECs.

\section{Discussion}

To the best of our knowledge, this is the first study to demonstrate increased expression and distinct localization of APJ in HSCs/HPCs associated with differentiated proliferative capillary ECs in early-stage NASH in humans.

Immature HSCs/HPCs have been shown to exit from circulation and lodge nearby the endothelium in experimental animals [7]. We demonstrated the expression of APJ in HSCs/ HPCs related to ECs and PCs in human liver.

Hypoxia is associated with the development and progress of NAFLD and lipid metabolism in the liver [8]. It has been well established that hypoxia/ischemia triggers $\mathrm{HSCs} / \mathrm{HPCs}$ to migrate from the bone marrow into peripheral blood [9]. After migration to the site of hypoxic/ischemic tissue, HSCs/HPCs can differentiate into ECs and participate in the formation of novel vessels. HIF- $1 \alpha$ is a key determinant of oxygen-dependent gene regulation in angiogenesis, which has been shown to be involved in HSC/HPC proliferation and differentiation, through low oxygen tension (hypoxia), which has been termed "hypoxic niche" [9]. HIF-1 $\alpha$, as well as its downstream apelin/ APJ signaling, were upregulated in hypoxia-treated HPCs, and might therefore serve as a potential target for the prevention of hypoxic ischemic injury in vitro [10]. In sprouting angiogenesis, angiopoietin-1 and apelin function as important factors that support mature ECs sprouting from pre-existing vessels [4]. APJ was highly expressed in HSCs and hepatocytes in cirrhotic liver, suggesting that hypoxia and inflammatory factors could play major roles in the activation of the hepatic apelin system, which can lead to angiogenic and fibroproliferative responses in chronic liver disease [11].

In conclusion, HSCs/HPCs expressing APJ may contribute to the angiogenesis of liver tissue in early-stage NASH.

\section{Acknowledgments}

The authors thank Hitoshi Yamazaki and Yoshihito Takahashi of Kitasato University Medical Center. The authors also thank Mariko Ogi and Tomoko Yoshii for technical assistance.

\section{Author Contributions}

HY, KO and MO planned the study. HY and WA conducted the 


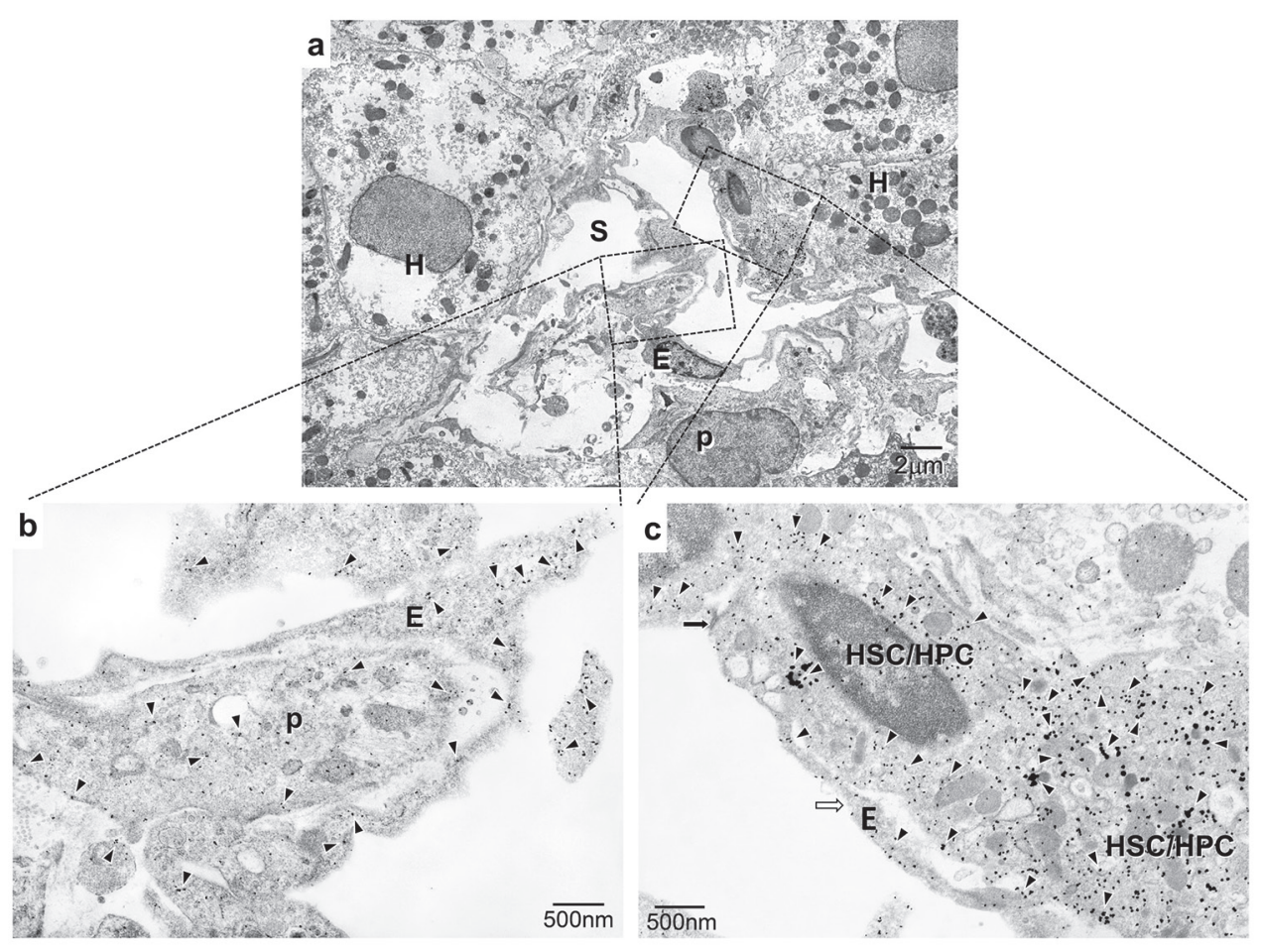

Figure 2. Immunoelectron microscopic observation of APJ in early NASH liver. (a) APJ expression is aberrantly observed at the proliferated arterial capillaries opening into the sinusoid. (b) APJ expression is localized at proliferated capillary ECs and pericytes. (c) APJ expression is localized at HSCs/HPCs. S: hepatic sinusoid; EC: endothelial cell; H: hepatocyte; p: pericyte; HSC/ HPC: hematopoietic stem cell/progenitor cell. (a) Low magnification. (b, c) High magnification.

experiments. WA and HY wrote the manuscript.

\section{Abbreviations}

APJ: apelin receptor; EC: endothelial cell; HSC/HPC: hematopoietic stem cell/progenitor cell; IEM: immunoelectron microscopy; IHC: immunohistochemistry; NAFLD: non-alcoholic fatty liver disease; NASH: non-alcoholic steatohepatitis; PC: pericyte

\section{References}

1. Risau W. Mechanisms of angiogenesis. Nature. 1997;386(6626):671-674.

2. Tatemoto K, Hosoya M, Habata Y, Fujii R, Kakegawa T, Zou MX, Kawamata Y, et al. Isolation and characterization of a novel endogenous peptide ligand for the human APJ receptor. Biochem Biophys Res Commun. 1998;251(2):471-476.

3. O'Carroll AM, Lolait SJ, Harris LE, Pope GR. The apelin receptor APJ: journey from an orphan to a multifaceted regulator of homeostasis. J Endocrinol. 2013;219(1):R1335.

4. Takakura N, Watanabe T, Suenobu S, Yamada Y, Noda T, Ito Y, Satake M, et al. A role for hematopoietic stem cells in promoting angiogenesis. Cell. 2000;102(2):199-209.
5. Brunt EM, Janney CG, Di Bisceglie AM, NeuschwanderTetri BA, Bacon BR. Nonalcoholic steatohepatitis: a proposal for grading and staging the histological lesions. Am J Gastroenterol. 1999;94(9):2467-2474.

6. Kawanaka M, Nishino K, Nakamura J, Suehiro M, Goto D, Urata N, Oka T, et al. Treatment of nonalcoholic steatohepatitis with vitamins $\mathrm{E}$ and $\mathrm{C}$ : a pilot study. Hepat Med. 2013;5:11-16.

7. Tamplin OJ, Durand EM, Carr LA, Childs SJ, Hagedorn EJ, Li P, Yzaguirre AD, et al. Hematopoietic stem cell arrival triggers dynamic remodeling of the perivascular niche. Cell. 2015;160(1-2):241-252.

8. Suzuki T, Shinjo S, Arai T, Kanai M, Goda N. Hypoxia and fatty liver. World J Gastroenterol. 2014;20(41):1508715097.

9. Wang LD, Wagers AJ. Dynamic niches in the origination and differentiation of haematopoietic stem cells. Nat Rev Mol Cell Biol. 2011;12(10):643-655.

10. Zhang J, Liu Q, Hu X, Fang Z, Huang F, Tang L, Zhou $\mathrm{S}$. Apelin/APJ signaling promotes hypoxia-induced proliferation of endothelial progenitor cells via phosphoinositide-3 kinase/Akt signaling. Mol Med Rep. 2015;12(3):3829-3834.

11. Melgar-Lesmes P, Pauta M, Reichenbach V, Casals G, Ros J, Bataller R, Morales-Ruiz M, et al. Hypoxia and proinflammatory factors upregulate apelin receptor expression in human stellate cells and hepatocytes. Gut. 2011;60(10):1404-1411. 